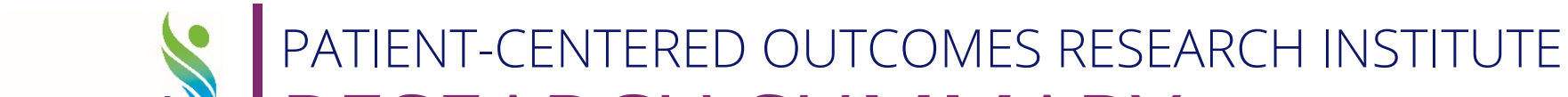 pcori?. RESEARCH SUMMARY
}

\section{Understanding the Development of Children with Cerebral Palsy and How Therapy May Affect Patient-Centered Outcomes}

Principal investigator

Sarah Westcott McCoy, PhD
Organization

University of Washington

\section{What was the research about?}

Cerebral palsy, or CP, is a health problem that starts at birth or in early childhood and affects the nerves and muscles. CP can reduce movement and endurance. Children with CP have different levels of functional ability, or the ability to do everyday activities. Children with CP also develop in different ways as they age.

In this study, therapists assessed more than 700 children with CP. The therapists did this two to five times over two years. The research team then made development charts, which showed how children with CP at different levels of functional ability may develop as they age. The team also looked at the therapy the children received, such as physical, occupational, and speech therapy. This therapy helps children with CP learn to move and do everyday activities. The team wanted to learn how different aspects of therapy were related to the children's endurance and their taking part in leisure and selfcare activities.

\section{What were the results?}

Development charts. Overall, the lower a child's functional ability, the lower the rate and amount of development. The charts show that for most measures, children develop quickly at young ages. Then their development slows.
Therapy. Children with lower functional ability had more therapy sessions. But more sessions didn't lead to better development.

Aspects of therapy related to better development in endurance and the ability to take part in leisure and self-care activities included therapy in which

- Therapists worked closely with the child's family

- Parents felt their child's therapy needs were being met

Aspects of therapy related to better development only in the ability to take part in leisure and self-care activities included therapy with a focus on

- Health and well-being

- Structured play and activities

\section{Who was in the study?}

The study included 708 children with CP. The children were between 18 months and 12 years old. They were from four regions in the United States and six regions in Canada.

\section{What did the research team do?}

Therapists looked at the children's balance, alignment of the spine and range of motion, strength, and 
endurance. Parents also filled out a form about their child's endurance, other health issues, and ability to take part in leisure and self-care activities. The research team made development charts for each of these measures. The charts showed the average development for children at different levels of functional ability as they aged.

Parents also filled out a form on the aspects of the therapy their child had received. The research team looked at how certain aspects of therapy were related to the children's endurance and their ability to take part in leisure and self-care activities.

Seven mothers of children with CP gave input during the study.

\section{What were the limits of the study?}

Information about therapy services came from parents, not the children's therapists. The study didn't look at how effective the services were. Also, the study didn't compare different services.

\section{How can people use the results?}

Families of children with CP, their doctors, and their therapists can use the results to understand how children with $\mathrm{CP}$ at different levels of functional ability may develop. However, the results cannot predict the progress of an individual child. Families and doctors can also use the results to talk about what therapy services may be helpful for children with CP.

To learn more about this project, visit pcori.org/McCoy065. 